\title{
Late Divergence of Target and Nontarget ERPs in a Visual Oddball Task
}

\author{
A. DAMBORSKÁ ${ }^{1,2}$, M. BRÁZDIL ${ }^{1,3}$, I. REKTOR ${ }^{1,3}$, E. JANOUŠOVÁ ${ }^{4}$, J. CHLÁDEK ${ }^{5}$, \\ M. KUKLETA ${ }^{1}$
}

${ }^{1}$ CEITEC - Central European Institute of Technology, Masaryk University, Brno, Czech Republic, ${ }^{2}$ Department of Physiology, Faculty of Medicine, Masaryk University, Brno, Czech Republic, ${ }^{3}$ First Department of Neurology, St. Anne's Faculty Hospital, Masaryk University, Brno, Czech Republic, ${ }^{4}$ Institute of Biostatistics and Analyses, Masaryk University, Brno, Czech Republic, ${ }^{5}$ Institute of Scientific Instruments, Academy of Sciences of the Czech Republic, Brno, Czech Republic

Received June 24, 2011

Accepted January 26, 2012

On-line April 5, 2012

\section{Summary}

Different mental operations were expected in the late phase of intracerebral ERPs obtained in the visual oddball task with mental counting. Therefore we searched for late divergences of target and nontarget ERPs followed by components exceeding the temporal window of the P300 wave. Electrical activity from 152 brain regions of 14 epileptic patients was recorded by means of depth electrodes. Average target and nontarget records from 1800 ms long EEG periods free of epileptic activity were compared. Late divergence preceded by almost identical course of the target and nontarget ERPs was found in 16 brain regions of 6 patients. The mean latency of the divergence point was $570 \pm 93 \mathrm{~ms}$ after the stimulus onset. The target post-divergence section of the ERP differed from the nontarget one by opposite polarity, different latency of the components, or even different number of the components. Generators of post-divergence ERP components were found in the parahippocampal gyrus, superior, middle and inferior temporal gyri, amygdala, and fronto-orbital cortex. Finding of late divergence indicates that functional differences exist even not sooner than during the final phase of the task.

\section{Key words}

Intracerebral recording • Oddball task • Visual evoked potentials - Mental counting • Memory

\section{Corresponding author}

Alena Damborská, Department of Physiology, Faculty of Medicine, Masaryk University, Kamenice 5, CZ-625 00 Brno, Czech Republic. E-mail: adambor@med.muni.cz

\section{Introduction}

Scalp-recorded event-related potentials (ERPs) elicited during the so-called "oddball" task have been employed for decades as a useful tool for studying the processes of cognition. In the oddball task the subject responds by button pressing and/or mental counting only to the infrequent "target" (T) stimulus, which is presented randomly and repeatedly among frequent "nontarget" (NT) stimuli. The electrophysiological response to the targets is compared with that to the nontargets and the difference is taken as a measure of the differences in underlying brain processes (Sutton et al. 1965). As a rule, these ERPs are composed of several components, which differ in amplitude, latency, and/or polarity. The early or exogenous ERP components are thought to express the stimulus identification processes (Jewett et al. 1970, Grönfors 1993). On scalp recording these components are referred to as $\mathrm{P} 100, \mathrm{~N} 100$, and $\mathrm{P} 200$. The later or endogenous ERP components are considered to be associated with cognitive processes. One of the most studied endogenous ERP components is the P300 or P3 wave, the largest positive-going peak occurring after the exogenous components within a time window of 250-500 ms. Subsequent research showed that this component occurs in two main variants, P3a and P3b (Polich 1998, Comerchero and Polich 1999). These variants differ in latency and amplitude distribution. 
According to recent interpretation (Polich 2007), the $\mathrm{P} 3 \mathrm{a}$ originates from stimulus/driven frontal attention mechanisms during task processing, whereas P3b originates from temporal-parietal activity associated with attention and appears related to subsequent memory processing. It has also been suggested that the P300 may be related to updating internal models about context and environment, which is triggered by eventrelated changes in theta rhythms reflecting self-motion (Shin 2011), thus supporting the view that cognition may be tightly interlocked to motor activity.

The assessment of differences between the target and nontarget ERPs is usually based on the amplitude and latency differences in the components. The differences between the $\mathrm{T}$ and NT responses occur in exogenous components if the triggering stimulus situation is complex (Luck and Hillyard 1994). On the other hand, the P300 components always differ in the T and NT responses representing a key differential sign (Hillyard et al. 1971, O’Donnell et al. 1997). In the nontarget response the P300 component either does not appear at all or exhibits smaller amplitude (Kok 1997).

From the behavioral point of view the oddball task consists of a sequence of brain operations, which include the detection of the stimulus, decoding its significance, decision "what to do", execution of the instructed movement, and/or counting the T stimuli. The difference between the $\mathrm{T}$ and NT tasks in principle consists in motor response and counting of stimuli in the target trials and doing nothing in the nontarget ones. Searching for the spatiotemporal relationship between these operations using registration of the ERP is in scalp recording difficult because of large summation of the electrophysiological signals. The intracerebral recording is far more suitable in this respect. For example, in a number of cortical and subcortical brain structures generators of the P3-like wave were found using intracerebral electrodes (McCarthy et al. 1989, Baudena et al. 1995, Halgren et al. 1995a, b, 1998, Brázdil et al. 1999, 2003, Rektor et al. 2003, 2005, 2007, Sochůrková et al. 2006, Damborská et al. 2009). Intracerebral recording disclosed also brain sites with different temporal characteristics of the P3-like component in the visual oddball task: one type related to the stimulus, other type related to the movement, and yet another type without any obvious relationship to these events (Roman et al. 2005). Another study reports on the modality specific P3-like component elicited in certain brain sites (Halgren et al. 1995b). With the help of intracerebral recording, generators of late evoked potentials similar to P3-like component were also found in Contingent Negative Variation paradigm in cortical as well as subcortical sites (Bareš et al. 2003). Intracerebral recording methods were also employed in studies using different response conditions in which the existence of task-specific P3-like potential generators (Brázdil et al. 2003) and of Contingent Negative Potential generators (Bareš et al. 2007) was proved.

The description of spatiotemporal characteristics of intracerebral ERP components may provide relevant data for understanding of organization and functional principles of cognitive networks (Brázdil et al. 2003, Kukleta et al. 2003, Roman et al. 2005, Damborská et al. 2006, Rektor et al. 2007). The oddball paradigm seems to be very useful in this respect, as it consists of sequence of partial functions both in target and nontarget variants. These functions are believed to be associated with the sequence of ERP components and thus the point of divergence (DP) between the target and nontarget ERPs found in a given brain site can be considered as a demonstration of the beginning of a functional divergence. In accordance with this interpretation the onset of the P3-like component could be considered as one example of such divergences. While there are many intracerebral studies aimed at the P3-like component in the visual oddball task, only two of them concerned also components of longer latency. In lateralized visual oddball intracerebral studies (Clarke et al. 1999a, b) patients exhibited late (>600 ms peak latency) ERP components with slow/broad morphology in response to target stimuli that, in turn, were either absent or of smaller amplitude to nontarget stimuli. These components were typically negativegoing and followed behavioral motor responses. They were pervasive, and polarity reversals were present in the insula/operculum region. Authors interpreted them as reflecting activity from secondary somatosensory cortex.

In the current study we searched for the divergence points of the target and nontarget ERPs in very late phase of the visual oddball task with mental counting. We supposed that even in the time window exceeding the latency of the P3-like component, differences between the target and nontarget ERPs should appear, thus giving rise to such late divergences, because different mental operations are expected in this phase. 
Table 1. Investigated brain structures.

\begin{tabular}{|c|c|c|c|}
\hline Patient & $\begin{array}{l}\text { Electrodes } \\
\text { (left/right) }\end{array}$ & $\begin{array}{l}\text { Contacts } \\
\text { (left/right) }\end{array}$ & Structures \\
\hline 1 & $5 / 2$ & $28 / 13$ & $\begin{array}{l}\text { AMY', } \mathrm{PHG}^{\prime}, \mathrm{FG}^{\prime}, \mathrm{STG}^{\prime}, \mathrm{MTG}^{\prime}, \mathrm{ITG}^{\prime}, \mathrm{FOC}^{\prime}, \mathrm{RG}^{\prime} \\
\text { PHG, ITG, CG, A6 }\end{array}$ \\
\hline 2 & $5 / 4$ & $25 / 16$ & $\begin{array}{l}\text { AMY', HIP', } \text { PHG }^{\prime}, \mathrm{STG}^{\prime}, \mathrm{MTG}^{\prime}, \mathrm{ITG}^{\prime}, \mathrm{OG}^{\prime} \\
\text { AMY, HIP, PHG, MTG, OG }\end{array}$ \\
\hline 3 & $4 / 1$ & $27 / 15$ & $\begin{array}{l}\mathrm{AMY}^{\prime}, \mathrm{PHG}^{\prime}, \mathrm{FG}^{\prime}, \mathrm{MTG}^{\prime}, \mathrm{ITG}^{\prime}, \mathrm{CG}^{\prime}, \mathrm{DLPFC}^{\prime}, \mathrm{WTL}^{\prime} \\
\text { HIP, BG, WFL }\end{array}$ \\
\hline 4 & $1 / 6$ & $15 / 26$ & 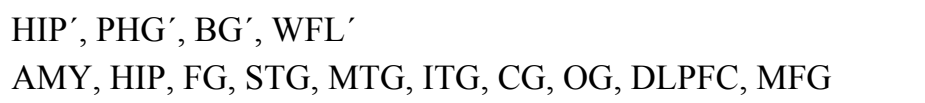 \\
\hline 5 & $1 / 1$ & $14 / 14$ & $\begin{array}{l}\mathrm{AMY}^{\prime}, \mathrm{HIP}^{\prime}, \mathrm{BG}^{\prime}, \mathrm{WTL}^{\prime}, \mathrm{WFL}^{\prime} \\
\text { AMY, HIP, BG, WTL, WFL }\end{array}$ \\
\hline 6 & $6 / 4$ & $24 / 17$ & 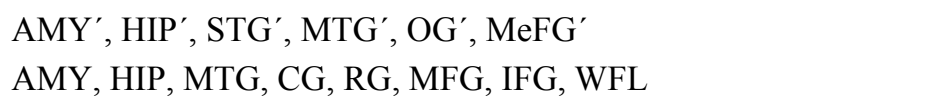 \\
\hline 7 & $1 / 3$ & $12 / 29$ & $\begin{array}{l}\text { AMY', HIP' }^{\prime} \\
\text { HIP, PHG, STG, DLPFC, FOC }\end{array}$ \\
\hline 8 & $1 / 3$ & $13 / 28$ & $\begin{array}{l}\mathrm{AMY}^{\prime}, \mathrm{PHG}^{\prime}, \mathrm{BG}^{\prime}, \mathrm{STG}^{\prime}, \mathrm{MTG}^{\prime}, \mathrm{WTL}^{\prime}, \mathrm{WFL}^{\prime} \\
\text { AMY, HIP, BG, STG, MTG, ITG, WTL, WFL }\end{array}$ \\
\hline 9 & $0 / 6$ & $0 / 41$ & AMY, HIP, PHG, FG, STG, MTG, ITG, FOC, RG, MeFG, MFG \\
\hline 10 & $0 / 4$ & $0 / 27$ & AMY, HIP, STG, MTG, WTL \\
\hline 11 & $0 / 6$ & $0 / 36$ & HIP, FG, STG, MTG, ITG, CG, A4, A5, IPL, MT, WTL, WPL \\
\hline 12 & $4 / 5$ & $15 / 26$ & $\begin{array}{l}\mathrm{HIP}^{\prime}, \mathrm{MTG}^{\prime}, \mathrm{CG}^{\prime}, \mathrm{DLPFC}{ }^{\prime}, \mathrm{A} 8^{\prime}, \mathrm{A} 9^{\prime}, \mathrm{A} 10^{\prime} \\
\text { HIP, MTG, CG, DLPFC, MFG, A8, WTL, WFL }\end{array}$ \\
\hline 13 & $7 / 0$ & $41 / 0$ & $\mathrm{AMY}^{\prime}, \mathrm{HIP}^{\prime}, \mathrm{STG}^{\prime}, \mathrm{MTG}^{\prime}, \mathrm{CG}^{\prime}, \mathrm{DLPFC}^{\prime}, \mathrm{FOC}^{\prime}, \mathrm{A}^{\prime}, \mathrm{WFL}^{\prime}$ \\
\hline 14 & $3 / 0$ & $29 / 0$ & $\mathrm{AMY}^{\prime}, \mathrm{PHG}^{\prime}, \mathrm{FG}^{\prime}, \mathrm{BG}^{\prime}, \mathrm{MTG}^{\prime}, \mathrm{ITG}^{\prime}, \mathrm{WTL} \mathrm{L}^{\prime}$ \\
\hline
\end{tabular}

Number of the patient; number of electrodes implanted in the left and right hemispheres; number of contacts exploring sites in the left and right hemispheres; anatomical structures investigated: AMY - amygdala, HIP - hippocampus, PHG - parahippocampal gyrus, FG fusiform gyrus, BG - basal ganglia, STG, MTG, and ITG - superior, middle, and inferior temporal gyri, CG - cingulate gyrus, DLPFC dorsolateral prefrontal cortex, FOC - fronto-orbital cortex, OG - orbital gyri, RG - rectal gyrus, MeFG, MFG, and IFG - medial, middle, and inferior frontal gyri, A 4, 5, 6, 8, 9, and 10 - Brodmann's areas 4, 5, 6, 8, 9, and 10, IPL - inferior parietal lobule, MT mesencephalic tegmentum, WTL, WFL, and WPL - white matter of the temporal, frontal, and parietal lobes, symbol (') indicates structures of the left hemisphere.

\section{Materials and Methods}

\section{Subjects}

Fourteen patients ( 3 women) aged from 20 to 45 years were employed in the study. All subjects suffered from medically intractable epilepsy and were candidates for surgical treatment. They all were under antiepileptic drug therapy, which was determined by clinical considerations. During the period of diagnostic examination by intracerebral EEG recording, the doses of medicaments were reduced to allow seizures to develop spontaneously. They had normal or corrected-to-normal vision and all but one (Patient No. 6) were right-handed. The subjects gave us their informed consent to the experimental protocol that had been approved by the Ethical Committee of Masaryk University.

\section{Paradigm}

A visual oddball task with mental counting was performed. The patients were sitting comfortably in a moderately lighted room and were focusing the centre of a monitor situated at about $100 \mathrm{~cm}$ from their eyes. Yellow capital letters X (target, T) or O (nontarget, NT) appeared repeatedly on white background in random order as experimental stimuli. Each stimulus presentation lasted $200 \mathrm{~ms}$ and the interstimulus interval varied randomly between 2 and 5 seconds. The target stimuli were five times less frequent than the nontarget ones. The 
subjects were instructed to press a microswitch button with the dominant hand as quickly as possible, whenever a $\mathrm{T}$ stimulus appeared, to mentally count the $\mathrm{T}$ stimuli, and to ignore the NT stimuli.

\section{Data acquisition}

Electrical activity was recorded during the task simultaneously from various brain sites by means of standard Micro Deep semi-flexible multicontact platinum electrodes. Having a diameter of $0.8 \mathrm{~mm}$, each electrode carried 5-15 contacts $2.0 \mathrm{~mm}$ long separated by constant intervals of $1.5 \mathrm{~mm}$. Strictly for diagnostic reasons, intracerebral depth electrodes were implanted to the patients; and structures of the frontal, temporal, and parietal lobes were examined (Table 1). Every patient received from 2 to 10 such electrodes exploring either or both hemispheres. Long electrodes examined both lateral and mesial cortical regions. The electrodes were placed using the methodology of Talairach et al. (1967) and their position was afterwards verified by magnetic resonance imaging with electrodes in situ. The registration was made with the help of a 64-channel Brain Quick EEG system (Micromed). All the recordings were monopolar with respect to a reference electrode attached to the right processus mastoideus. The impedances used were less than $5 \mathrm{k} \Omega$. The EEG signal was amplified with a bandwidth of $0.1-40 \mathrm{~Hz}$ at a sampling rate of $128 \mathrm{~Hz}$. One of the channels recorded the button pressing and yet another channel recorded the presentation of the experimental stimuli. We did not do electrooculography, because in contrast to the scalp recordings artefacts caused by eye movements and blinking are considered to be negligible in depth recordings.

\section{Analysis}

The signal analysis was made offline with the help of ScopeWin software providing us 44 channels for simultaneous recordings. The recordings from lesions and epileptogenic zones and the trials with artefacts were rejected offline with visual inspection made by experienced person. Switching the button in response to a nontarget stimulus or its omission in response to a target one were considered as errors. In each subject all artefactfree trials with correct responses were used for calculation of average curves. Excluding of different number of trials explains the interindividual variability in number of trials used (29-58 T trials, 198-331 NT trials). Peristimulus EEG periods (from -300 to $+1500 \mathrm{~ms}$ from the stimulus onset) were averaged separately for $\mathrm{T}$ and
NT responses using the stimulus onset as a trigger. The statistical significance of ERP components was computed between the mean amplitude observed during the baseline region (from -600 to $-100 \mathrm{~ms}$ from the stimulus onset) and the mean value computed as a mean from the neighbourhood of each point (170 ms length) after stimuli using a non-parametric Wilcoxon Rank Sum (Signed Rank) test for paired samples.

Records from one contact of each multicontact intracerebral electrode implanted in a particular anatomical structure were included into the analysis selecting the largest one from ERPs. In such selected contacts the amplitude differences between the target and nontarget records were assessed using a cluster-based permutation test (Maris and Oostenveld 2007). In every contact, clusters of time samples whose absolute t-value was larger than 97.5th quantile of T-distribution were computed in the poststimulus EEG period (from $0 \mathrm{~ms}$ to $+1500 \mathrm{~ms}$ from the stimulus onset). Monte-Carlo estimates of the permutation p-values for each cluster were calculated on 1000 random partitions of the data set and compared with a critical alpha-level of 0.05 . We disclosed brain regions with an initially almost identical course of the target and nontarget ERPs followed by a clear-cut divergence. The onset of such divergence was identified as the starting time point of the first statistically significant cluster in the post-stimulus period. To assess the portion of ERPs, attributable to movement-related potentials, we investigated the character of the relationship between the latency of a particular target post-divergence ERP component and the reaction time using the method already applied for classification of P3like waves (Roman et al. 2005).

\section{Results}

The performance of subjects during the task was very good not seeming to be substantially influenced by their illness and medication (only $1.3 \pm 1.7 \%$ of all responses were incorrect; mean patient's SRI varied between $457 \pm 34 \mathrm{~ms}$ and $644 \pm 78 \mathrm{~ms}$, median $525 \pm 63 \mathrm{~ms}$ ). Total number of explored brain regions was 152; ERPs were found in 102 regions, which make $67 \%$ of explored regions. In assessing the number of ERP components, their polarity, latency and amplitude, various regions generating several types of ERP were identified: 1) regions that generated different ERPs to target and nontarget stimuli (22 brain regions); 2) regions that generated the target and nontarget ERPs exhibiting clearcut divergence after initially almost identical curves 
(41 brain regions); 3) regions that generated target and nontarget ERPs with almost identical course but statistically significantly different amplitudes in some of their components (20 brain regions); 4) regions generating ERP only to target but not to nontarget stimuli (9 brain regions); 5) regions generating almost identical ERPs to target and nontarget stimuli (10 brain regions).

In accordance with the aim of the current study, only ERPs from group 2) were further analyzed. In this group ERPs were arbitrarily divided into two subgroups according to the latency of their divergence. The first subgroup (early divergence) included ERPs diverging sooner than $420 \mathrm{~ms}$ after the stimulus onset and the second subgroup (late divergence) included ERPs diverging later than $420 \mathrm{~ms}$ after the stimulus onset (see Figure 1 and Table 2).

ERPs exhibiting the early divergence were found in nine patients in 25 brain regions (15 in the frontal lobe, 8 in the temporal lobe, and 2 in the parietal lobe). The mean latency of the point of divergence was $346 \pm 35 \mathrm{~ms}$ from the stimulus onset. ERPs exhibiting the late divergence were found in six patients in 16 brain regions (13 in the temporal lobe and 3 in the frontal lobe). The mean latency of the point of divergence was $570 \pm 93 \mathrm{~ms}$ from the stimulus onset. Statistical testing showed significant amplitude differences in post-divergence section of all the early and late diverging ERPs (clusterbased permutation test).

Table 2. Early and late divergences of the target and nontarget ERPs.

\begin{tabular}{|c|c|c|c|c|c|c|c|}
\hline \multicolumn{4}{|c|}{ EARLY } & \multicolumn{4}{|c|}{ LATE } \\
\hline \multirow{2}{*}{$\begin{array}{l}\text { Patient } \\
\text { (Contact) }\end{array}$} & \multirow[t]{2}{*}{ Struct. } & \multicolumn{2}{|c|}{ Latency } & \multirow{2}{*}{$\begin{array}{l}\text { Patient } \\
\text { (Contact) }\end{array}$} & \multirow[t]{2}{*}{ Struct. } & \multicolumn{2}{|c|}{ Latency } \\
\hline & & ms & $\%$ RT & & & ms & $\%$ RT \\
\hline $1\left(C^{\prime} 6\right)$ & ITG & 280 & 49 & $1\left(A^{\prime} 1\right)$ & AMY & 440 & 77 \\
\hline $1(F 3)$ & $\mathrm{CG}$ & 360 & 63 & $1\left(B^{\prime} 2\right)$ & PHG & 536 & 93 \\
\hline $1(F 11)$ & Area 6 & 368 & 64 & $1(B 2)$ & PHG & 568 & 98 \\
\hline $1\left(O^{\prime} 2\right)$ & FOC & 320 & 56 & $1\left(C^{\prime} 2\right)$ & PHG & 640 & 111 \\
\hline $3\left(G^{\prime} 2\right)$ & $\mathrm{CG}$ & 312 & 66 & $1\left(T^{\prime} 3\right)$ & STG & 640 & 111 \\
\hline $4(G 1)$ & $\mathrm{CG}$ & 352 & 67 & $2\left(B^{\prime} 5\right)$ & ITG & 616 & 96 \\
\hline $5(X 14)$ & $\mathrm{BG}$ & 312 & 55 & $2\left(C^{\prime} 2\right)$ & PHG & 488 & 76 \\
\hline $6\left(D^{\prime} 4\right)$ & STG & 344 & 68 & $2(C 3)$ & $\mathrm{FG}$ & 552 & 86 \\
\hline $6(G 2)$ & $\mathrm{CG}$ & 392 & 78 & $3\left(C^{\prime} 14\right)$ & MTG & 448 & 95 \\
\hline $6\left(O^{\prime} 6\right)$ & $\mathrm{OG}$ & 376 & 75 & $3\left(B^{\prime} 3\right)$ & PHG & 800 & 169 \\
\hline $6(06)$ & IFG & 416 & 83 & $4(A 9)$ & MTG & 664 & 126 \\
\hline $6\left(T^{\prime} 1\right)$ & STG & 368 & 73 & $4(C 10)$ & ITG & 624 & 119 \\
\hline $6(B 9)$ & MTG & 328 & 65 & $4\left(X^{\prime} 1\right)$ & PHG & 592 & 112 \\
\hline $6(G 10)$ & MFG & 336 & 67 & $5\left(X^{\prime} 10\right)$ & BG & 544 & 95 \\
\hline $11(B 4)$ & HIP & 320 & 67 & $9(011)$ & FOC & 520 & 89 \\
\hline $11(B 9)$ & MTG & 344 & 72 & $9(P 1)$ & MeFG & 440 & 75 \\
\hline $11(G 2)$ & $\mathrm{CG}$ & 312 & 65 & & & & \\
\hline $11(R 5)$ & Area 5 & 344 & 72 & & & & \\
\hline $11(B 14)$ & ITG & 408 & 85 & & & & \\
\hline $11(G 11)$ & IPL & 376 & 79 & & & & \\
\hline $11(T 3)$ & STG & 400 & 84 & & & & \\
\hline $12\left(F^{\prime} 3\right)$ & Area 8 & 360 & 61 & & & & \\
\hline $13\left(G^{\prime} 1\right)$ & $\mathrm{CG}$ & 288 & 56 & & & & \\
\hline $13\left(O^{\prime} 8\right)$ & DLPFC & 312 & 61 & & & & \\
\hline $14\left(X^{\prime} 15\right)$ & $\mathrm{BG}$ & 328 & 72 & & & & \\
\hline
\end{tabular}

MFG - middle frontal gyrus, MeFG - medial frontal gyrus, IFG - inferior frontal gyrus, FOC - fronto-orbital cortex, OG - orbital gyri, DLPFC - dorsolateral prefrontal cortex, STG - superior temporal gyrus, MTG - middle temporal gyrus, ITG - inferior temporal gyrus; IPL - inferior parietal lobule, CG - cingulate gyrus; BG - basal ganglia, AMY - amygdala, FG - fusiform gyrus, PHG - parahippocampal gyrus, HIP - hippocampus, Area 5, Area 6, Area 8 - Brodmann's areas; symbol (') indicates structures of the left hemisphere, the divergence point latency is given in $\mathrm{ms}$ and in \% of patient's mean reaction time (\% RT). 


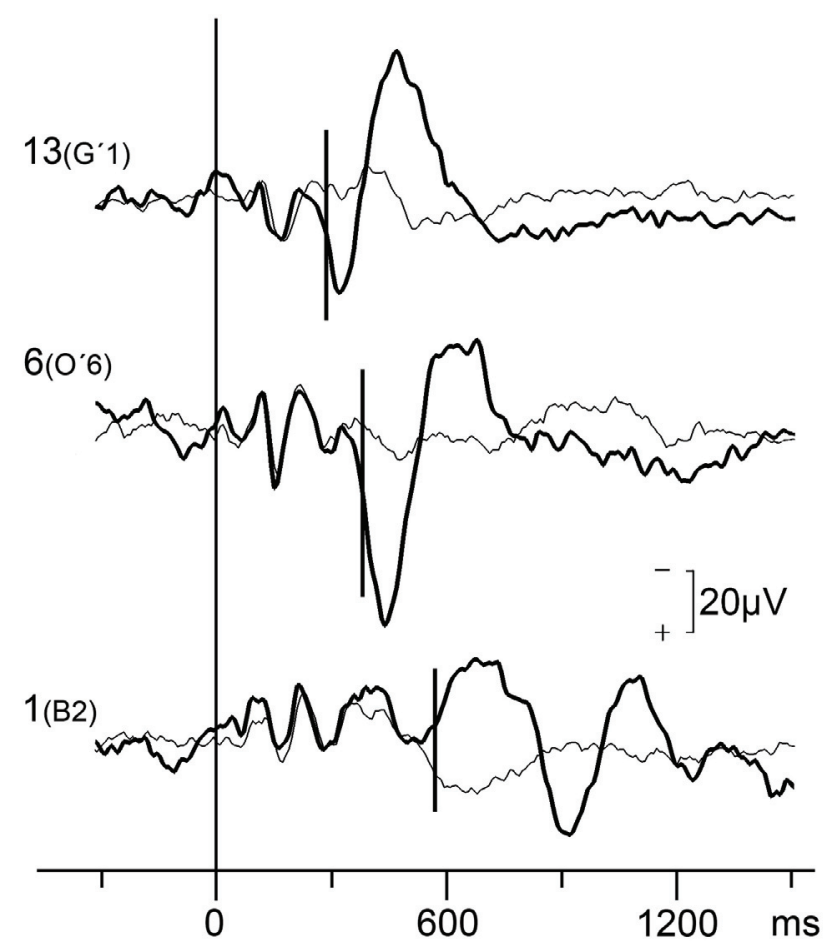

Fig. 1. Selected examples of early (288 ms and $376 \mathrm{~ms}$ ), and late (568 ms) divergences of ERPs elicited in response to target (thick curve) and nontarget (thin curve) stimuli. Curves are labelled with patient number and recording contact (13G'1 cingulate gyrus, $60^{\prime} 6$ - orbital gyri, $1 \mathrm{~B} 2$ - parahippocampal gyrus); vertical line at zero point indicates the stimulus onset; short vertical lines indicate the divergence points of target and nontarget ERPs (i.e. the starting time point of the first statistically significant cluster calculated in the post-stimulus period using the cluster-based permutation test); symbol (') indicates structures of the left hemisphere.

The relationship between the divergence point latency and the reaction time can be assessed from the values presented in the Table 2 . It is evident that the late divergence appeared shortly before, after, or approximately at the moment of the button pressing (in average at $102 \pm 23 \%$ of the mean reaction time).

Figure 2 shows all of the late diverging ERPs. Six of them were found in the right and ten of them in the left hemispheres. No clear-cut preponderance of ERPs exhibiting the late divergence was observed in any anatomical structure; however, most of these ERPs exhibited signs of local generation of post-divergence components in structures of temporal lobe. One example of local generation for each type of response is presented in Figure 3. Phase reversals of target post-divergence components were found in the amygdala (1A'1), frontoorbital cortex (9 O11), and parahippocampal gyrus (1B2); phase reversals of nontarget components were found in the amygdala (1A'1), parahippocampal gyrus (1B2), and middle temporal gyrus (4A9). Relative decrease of amplitude by $36 \pm 16 \%$ in adjacent contacts of the same electrode was observed on target post-divergence components in the parahippocampal gyrus (1B'2, 1C'2, $2 \mathrm{C}^{\prime} 2,3 \mathrm{~B}^{\prime} 3$, and $4 \mathrm{X}^{\prime} 1$ ), superior (1T'3), middle (3C'14 and 4A9), and inferior (2B'5 and 4C10) temporal gyri. Relative decrease of amplitude by $39 \pm 13 \%$ in adjacent contacts of the same electrode was observed on nontarget post-divergence components in the parahippocampal gyrus (1B'2, 1C'2, 2C'2, and 4X'1), middle (3C'14) and inferior (2B' 5 and $4 \mathrm{C} 10$ ) temporal gyri. Thus, generators of the target late post-divergence components were found in the amygdala (in 1 out of 16 explored regions), parahippocampal gyrus (in 6 out of 10 explored brain regions), fronto-orbital cortex (in 1 out of 4 explored brain regions), superior temporal gyrus (in 1 out of 11 explored brain regions), middle temporal gyrus (in 2 out of 16 explored brain regions), and inferior temporal gyrus (in 2 out of 9 explored brain regions), generators of the nontarget late post-divergence components were found in the same anatomical structures with the exception of the superior temporal and the fronto-orbital cortex.

From the records presented in Fig. 2 it is evident that clear-cut components were observed in the postdivergence section of not only all target but also of most nontarget late diverging ERPs. The post-divergence section of the target ERP was unequivocally different from the nontarget one exhibiting opposite polarity, different latency, or even different number of the components (see Table 3). The peak latency of the first post-divergence component varied between 516 and $968 \mathrm{~ms}$ (mean $695 \pm 140 \mathrm{~ms}$ ) in the $\mathrm{T}$ response and between 576 and $1128 \mathrm{~ms}$ (mean $725 \pm 145 \mathrm{~ms}$ ) in the NT response. The number of post-divergence ERP components varied between one and three in the $T$ response and was significantly higher than the number of post-divergence ERP components in the NT response, which varied between none and two $(p<0.001$, Student's paired $t$-test). From 32 target post-divergence ERP components (Table 3 ) only two were time-locked to the button pressing (superior temporal gyrus, fusiform gyrus), seven were time-locked to the stimulus onset (amygdala, parahippocampal gyrus, superior temporal gyrus) and 23 had ambiguous relationship to both these events (amygdala, parahippocampal gyrus, fusiform gyrus, middle and inferior temporal gyri, basal ganglia, fronto-orbital cortex, and medial frontal gyrus). 

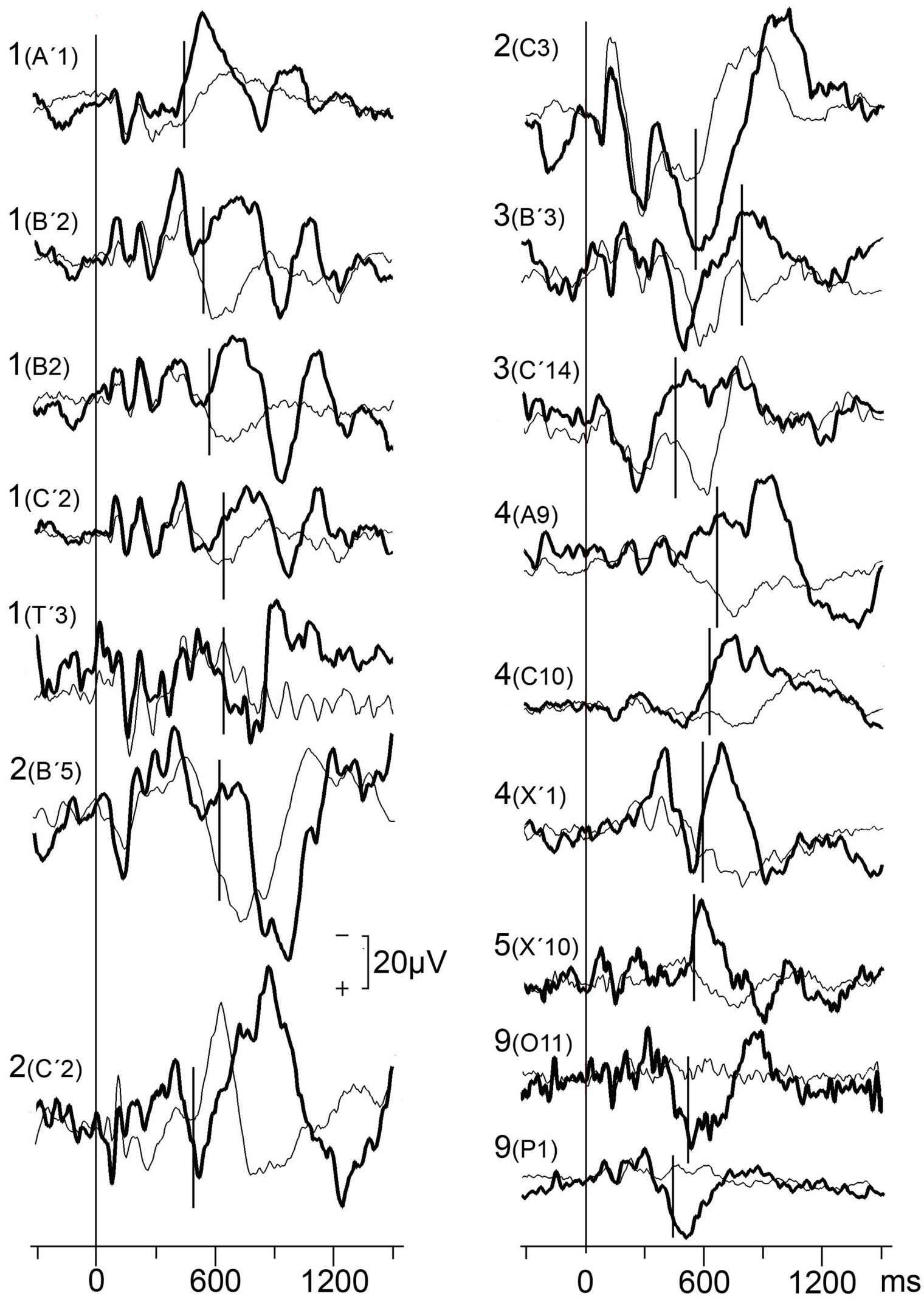

Fig. 2. Late divergence of the ERPs elicited in response to target (thick curve) and nontarget (thin curve) stimuli. Curves are labelled with patient number and recording contact; vertical line at zero point indicates the stimulus onset; short vertical lines indicate the divergence points of target and nontarget ERPs (i.e. the starting time point of the first statistically significant cluster calculated in the post-stimulus period using the cluster-based permutation test); symbol (') indicates structures of the left hemisphere. 
TARGET

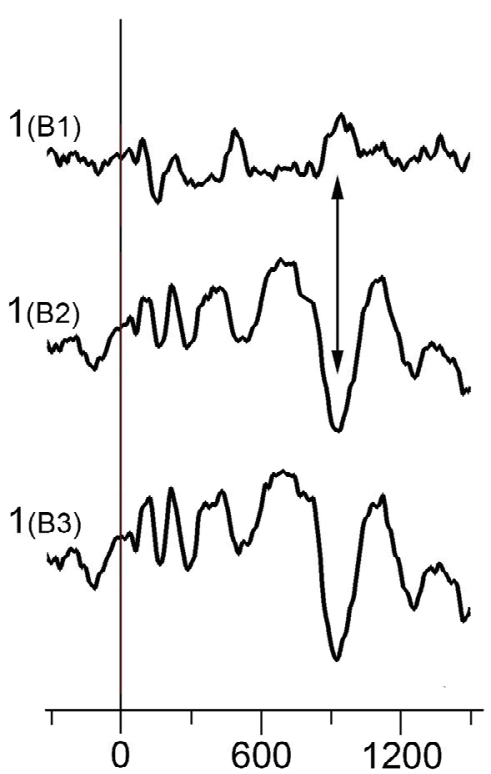

NONTARGET

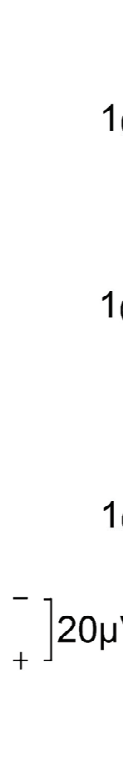

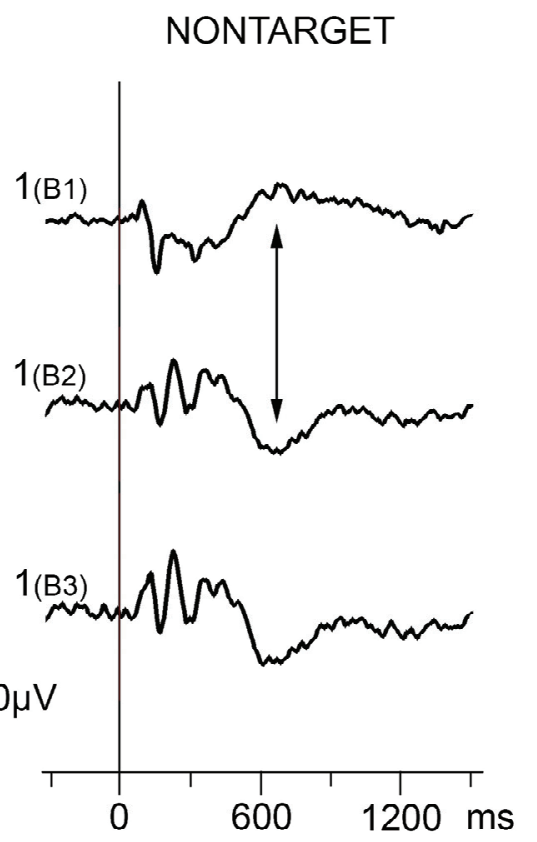

Fig. 3. An example of post-divergence ERP components. Left section: records from consecutive contacts of an electrode passing through the right parahippocampal gyrus (phase reversal between $B 1$ and $B 2$ ) in the target response; Right section: records from the same contacts in the nontarget response. Vertical line at zero point indicates the stimulus onset. Records from 1(B2) contact are also presented in Figure 1 and Figure 2.

Table 3. Polarity and latency of the target and nontarget late post-divergence components.

\begin{tabular}{|c|c|c|c|c|c|c|}
\hline \multirow{2}{*}{$\begin{array}{l}\text { Patient } \\
\text { (Contact) }\end{array}$} & \multirow{2}{*}{$\begin{array}{c}\text { Structure } \\
\text { AMY }\end{array}$} & \multicolumn{3}{|c|}{$\begin{array}{c}\text { Target } \\
\text { Post-divergence components }\end{array}$} & \multicolumn{2}{|c|}{$\begin{array}{c}\text { Nontarget } \\
\text { Post-divergence components }\end{array}$} \\
\hline & & $\mathrm{N} 528^{\mathrm{a}}$ & N992 & & N704 & \\
\hline $1\left(B^{\prime} 2\right)$ & PHG & N736 & P928 & $\mathrm{N} 1088^{\mathrm{a}}$ & P576 & \\
\hline $1(B 2)$ & PHG & N688 & P920 & $\mathrm{N} 1112^{\mathrm{a}}$ & P656 & \\
\hline $1\left(C^{\prime} 2\right)$ & PHG & N752 & P952 ${ }^{\mathrm{a}}$ & N1112 & P600 & N856 \\
\hline $1\left(T^{\prime} 3\right)$ & STG & P773 ${ }^{b}$ & N904 ${ }^{a}$ & & & \\
\hline $2\left(B^{\prime} 5\right)$ & ITG & P968 & & & P728 & N1072 \\
\hline $2\left(C^{\prime} 2\right)$ & PHG & P523 & N864 & P1240 & N600 & P776 \\
\hline $2(C 3)$ & FG & P568 ${ }^{b}$ & N944 & & N824 & \\
\hline $3\left(C^{\prime} 14\right)$ & MTG & N752 & & & P600 & N776 \\
\hline $3\left(B^{\prime} 3\right)$ & PHG & N828 & P1211 & & & \\
\hline $4(A 9)$ & MTG & N936 & P1360 & & P744 & \\
\hline $4(C 10)$ & ITG & N752 & & & N1128 & \\
\hline $4\left(X^{\prime} 1\right)$ & PHG & $\mathrm{N} 680^{\mathrm{a}}$ & N1200 & & P792 & \\
\hline $5\left(X^{\prime} 10\right)$ & $\mathrm{BG}$ & N584 & N882 & & P744 & \\
\hline $9(011)$ & FOC & P531 & N835 & & & \\
\hline $9(P 1)$ & $\mathrm{MeFG}$ & P516 & & & & \\
\hline
\end{tabular}

AMY - amygdala, PHG - parahippocampal gyrus, FOC - fronto-orbital cortex; STG - superior temporal gyrus, MTG - middle temporal gyrus, ITG - inferior temporal gyrus; FG - fusiform gyrus, BG - basal ganglia, MeFG - medial frontal gyrus, $\mathrm{N}$ - negativity, upward deflection, P - positivity, downward deflection; symbol (') indicates structures of the left hemisphere, ${ }^{a}$ component time-locked to the stimulus, ${ }^{b}$ component time-locked to the button pressing.

\section{Discussion}

Our results demonstrated the existence of the target and nontarget event-related potentials initially almost identical in course and then divergent, with the point of divergence manifesting in the very late phase of the visual oddball task. The question arose as to whether these late divergences could be related to the differences 
in motor demands between $\mathrm{T}$ and NT tasks. Certain factors suggest otherwise. The motor system activities that precede execution of movement include decisionmaking, action planning, and formation of a motor command. As the results of this study showed, the DP occurred shortly before, after, or at the moment of pressing the button. Thus, all of the pre-movement activities occurred during the period when the two EEG responses were following almost identical courses. Further, post-movement activity cannot be excluded from considerations of factors leading to the divergence. However, such involvement may only be partial because only negligible portion of ERP components was timelocked to the button pressing. The ERPs attributable to movement-related potentials were found in superior temporal and fusiform gyri.

Memory mechanisms are involved in the various steps of the task from beginning to end. They start with cognitive discrimination of the stimuli and continue with selection of the correct response, i.e. the decision as to whether to move or not and whether to count or not, in order to follow the instructions for the experiment. The next important step associated with memory processes involves counting the target stimuli. When counting, one must recall the result of the previous calculation, then do the calculation, which consists of adding "one" to the recalled number, and then store the new result in the memory.

Our results showed that majority of the generators of the late post-divergence ERP components were observed in structures known to participate in memory processes. Due to the fact that recording sites were selected according to diagnostic concerns, and many regions potentially engaged in the task were not explored, the possibility to use this result for functional interpretations is limited. Thus, it still remains in question as to whether the late post-divergence ERP components could represent mental counting processes or other processes, for instance those associated with so-called closure of the whole response (disengagement from the just finished decision, engagement to the consecutive one).

Our results also showed a predominance of targets over nontargets in terms of the number of late post-divergence ERP components. The higher number of late post-divergence ERP components indicated in the T variant suggests higher demands on memory functions in the $\mathrm{T}$ response. This interpretation is also in accordance with the results of the visual oddball studies without mental counting (Clarke et al. 1999a, b), where only single target components are reported in different brain sites during the very late phase of the task. Unlike in present study no counting process was performed after target stimulus presentation in these studies, which could led there to decreased number of late target ERP components.

In a paradigm where two different stimuli are presented and two different tasks are required it is not surprising that the ERPs diverge (for further details, see Brázdil et al. 2003, Rektor et al. 2007). Of more interest in the current study is the finding that the divergence point in several brain sites was observed so late in the course of the EEG response. In these responses, the targets and nontargets revealed an almost identical course of ERPs until this very late DP, thus suggesting that the stimuli were being processed equally in these brain sites for a very long time. It seems paradoxical that during the period, in which the stimuli have already been distinguished, while the subject must be aware of different demands and the motor response has already been planned and in some cases even executed, some sites in the brain keep responding almost identically. However, in the light of the previous demonstration of brain sites with task-relevant EEG activity almost identical during the whole oddball task (Kukleta et al. 2003); this finding does not appear so illogical. Evidently, as well as the specific electrical activities elicited in the oddball task, there are also activities that are common to the $\mathrm{T}$ and NT responses. As regards the processes underlying the non-specific activities, they could include consciousness and sustained attention, since these functions are required for both $\mathrm{T}$ and NT responses.

Extensive literature on event-related potentials in target detection tasks shows that the target and nontarget electrophysiological responses may diverge after sensory potentials had been elicited, giving rise to a postdivergence ERP component, well-known as the P300 component (see Polich 2007). This occurs after the initial phase of ERPs in which the stimulus is being detected and discriminated. This component represents the cognitive functions involved in the orientation of attention, contextual updating, response modulation, and response resolution. In scalp recordings it consists mainly of two variants, P3a and P3b (Courchesne et al. 1975, Squires et al. 1975, Knight 1984, Donchin and Coles 1988, Comerchero and Polich 1999, Polich 2007), which differ in their scalp topography and temporal 
characteristics. The P3a exhibits a frontal/central scalp distribution and a relatively short peak latency, while the P3b exhibits a parietal scalp distribution and relatively long peak latency. In this context the early postdivergence components presented in present study could correspond to the P3a-like and P3b-like waveforms.

The differences between EEG responses are generally viewed as reflecting differences between underlying functions engaged in the tasks. By the same logic, differences between the late post-divergence ERP components described in the current study (see Fig. 2 and Table 3) appear to embody different brain processes in the late phase of the target and nontarget task variants. These processes might be associated with the different closure of the task. It is not clear, however, to what extend these differences are associated with different demands on memory engagement in relation to the request for counting the target stimuli. These late postdivergence ERP components generated in the parahippocampal gyrus, superior, middle and inferior temporal gyri, amygdala, and fronto-orbital cortex represent a finding that was previously missing in the whole interpretation of ERP from visual oddball task with mental counting.

\section{Conflict of Interest}

There is no conflict of interest.

\section{Acknowledgements}

This work was supported by grants Nos. MSM0021622404, MUNI/A/0941/2010 and 1M06039 and the project "CEITEC - Central European Institute of Technology" (CZ.1.05/1.1.00/02.0068) from European Regional Development Fund. The technical part of the study was supported by grant P103/11/0933 of the Grant Agency of the CR. We wish to thank Mgr. Ladislav Červený and Tony Long for language help.

\section{References}

BAREŠ M, REKTOR I, KAŇOVSKÝ P, STREITOVÁ H: Cortical and subcortical distribution of middle and long latency auditory and visual evoked potentials in a cognitive (CNV) paradigm. Clin Neurophysiol 114: 2447 $2460,2003$.

BAREŠ M, NESTRAŠIL I, REKTOR I: The effect of response type (motor output versus mental counting) on the intracerebral distribution of the slow cortical potentials in an externally cued (CNV) paradigm. Brain Research Bulletin 71: 428-435, 2007.

BAUDENA P, HALGREN E, HEIT G, CLARKE JM: Intracerebral potentials to rare target and distracter auditory and visual stimuli. III. Frontal cortex. Electroencephalogr Clin Neurophysiol 94: 251-264, 1995.

BRÁZDIL M, REKTOR I, DUFEK M, DANIEL P, JURÁK P, KUBA R: The role of frontal and temporal lobes in visual discrimination task - depth ERP studies. Neurophysiol Clin 29: 339-350, 1999.

BRÁZDIL M, ROMAN R, DANIEL P, REKTOR I: Intracerebral somatosensory event-related potentials: effect of response type (button pressing versus mental counting) on P3-like potentials within the human brain. Clin Neurophysiol 114: 1489-1496, 2003.

CLARKE JM, HALGREN E, CHAUVEL P: Intracranial ERPs in humans during a lateralized visual oddball task: I. Occipital and peri-Rolandic recordings. Clin Neurophysiol 110: 1210-1225, 1999a.

CLARKE JM, HALGREN E, CHAUVEL P: Intracranial ERPs in humans during a lateralized visual oddball task: II. Temporal, parietal, and frontal recordings. Clin Neurophysiol 110: 1226-1244, $1999 \mathrm{~b}$.

COMERCHERO MD, POLICH J: P3a and P3b from typical auditory and visual stimuli. Clin Neurophysiol 110: 24-30, 1999.

COURCHESNE E, HILLYARD SA, GALAMBOS R: Stimulus novelty, task relevance and the visual evoked potential in man. Electroencephalogr Clin Neurophysiol 39: 131-143, 1975.

DAMBORSKÁ A, BRÁZDIL M, REKTOR I, ROMAN R, KUKLETA M: Correlation between stimulus-response intervals and peak amplitude latencies of visual P3 waves. Homeostasis Health Dis 44: 165-168, 2006.

DAMBORSKÁ A, BRÁZDIL M, REKTOR I, KUKLETA M: Temporal characteristics of intracerebral P3 wave in a visual oddball task. Physiol Res 58: 12P, 2009.

DONCHIN E, COLES MG: Is the P300 component a manifestation of context updating? Behav Brain Sci 11: 357-374, 1988. 
GRÖNFORS T: Identification of auditory brainstem responses. Int J Biomed Comput 32: 171-179, 1993.

HALGREN E, BAUDENA P, CLARKE JM, HEIT G, LIEGEOIS C, CHAUVEL P, MUSOLINO A: Intracerebral potentials to rare target and distracter auditory and visual stimuli. I. Superior temporal plane and parietal lobe. Electroencephalogr Clin Neurophysiol 94: 191-220, 1995a.

HALGREN E, BAUDENA P, CLARKE JM, HEIT G, MARINKOVIC K, DEVAUX B, VIGNAL JP, BIRABEN A: Intracerebral potentials to rare target and distracter auditory and visual stimuli. II. Medial, lateral and posterior temporal lobe. Electroencephalogr Clin Neurophysiol 94: 229-250, 1995 b.

HALGREN E, MARINKOVIC K, CHAUVEL P: Generators of the late cognitive potentials in auditory and visual oddball tasks. Electroencephalogr Clin Neurophysiol 106: 156-164, 1998.

HILLYARD SA, SQUIRES KC, BAUER JW, LINDSAY PH: Evoked potential correlates of auditory signal detection. Science 172: 1357-1360, 1971.

JEWETT DL, ROMANO MN, WILLISTON JS: Human auditory evoked potentials: possible brain stem components detected on the scalp. Science 167: 1517-1518, 1970.

KNIGHT RT: Decreased response to novel stimuli after prefrontal lesions in man. Electroencephalogr Clin Neurophysiol 59: 9-20, 1984.

KOK A: Event-related-potential (ERP) reflections of mental resources: a review and synthesis. Biol Psychol 45: 19-56, 1997.

KUKLETA M, BRÁZDIL M, ROMAN R, JURÁK P: Identical event-related potentials to target and frequent stimuli of visual oddball task recorded by intracerebral electrodes. Clin Neurophysiol 114: 1292-1297, 2003.

LUCK SJ, HILLYARD SA: Electrophysiological correlates of feature analysis during visual search. Psychophysiology 31: 291-308, 1994.

MARIS E, OOSTENVELD R: Nonparametric statistical testing of EEG- and MEG-data. J Neurosci Methods 164: $177-$ $190,2007$.

MCCARTHY G, WOOD CC, WILLIAMSON PD, SPENCER DD: Task-dependent field potentials in human hippocampal formation. J Neurosci 9: 4253-4268, 1989.

O'DONNELL BF, SWEARER JM, SMITH LT, HOKAMA H, MCCARLEY RW: A topographic study of ERPs elicited by visual feature discrimination. Brain Topogr 10: 133-143, 1997.

POLICH J: P300 clinical utility and control of variability. J Clin Neurophysiol 15: 14-33, 1998.

POLICH J: Updating P300: an integrative theory of P3a and P3b. Clin Neurophysiol 118: 2128-2148, 2007.

REKTOR I, KAŇOVSKÝ P, BAREŠ M, BRÁZDIL M, STREITOVÁ H, KLAJBLOVÁ H, KUBA R, DANIEL P: A SEEG study of ERP in motor and premotor cortices and in the basal ganglia. Clin Neurophysiol 114: 463471, 2003.

REKTOR I, BAREŠ M, BRÁZDIL M, KAŇOVSKÝ P, REKTOROVÁ I, SOCHŮRKOVÁ D, KUBOVÁ D, KUBA R, DANIEL P: Cognitive-and movement-related potentials recorded in the human basal ganglia. Mov Disord 20: 562-568, 2005.

REKTOR I, BRÁZDIL M, NESTRAŠIL I, BAREŠ M, DANIEL P: Modifications of cognitive and motor tasks affect the occurrence of event-related potentials in the human cortex. Eur J Neurosci 26: 1371-1380, 2007.

ROMAN R, BRÁZDIL M, JURÁK P, REKTOR I, KUKLETA M: Intracerebral P3-like waveforms and the length of the stimulus-response interval in a visual oddball paradigm. Clin Neurophysiol 116: 160-171, 2005.

SHIN J: The interrelationship between movement and cognition: theta rhythm and the P300 event-related potential. Hippocampus 21: 744-752, 2011.

SOCHŮRKOVÁ D, BRÁZDIL M, JURÁK P, REKTOR I: P3 and ERD/ERS in a visual oddball paradigm: a depth EEG study from the mesial temporal structures. J Psychophysiol 20: 32-39, 2006.

SQUIRES NK, SQUIRES KC, HILLYARD SA: Two varieties of long-latency positive waves evoked by unpredictable auditory stimuli in man. Electroencephalogr Clin Neurophysiol 38: 387-401, 1975.

SUTTON S, BAREN M, ZUBIN J, JOHN ER: Evoked-potential correlates of stimulus uncertainty. Science 150: $1187-$ $1188,1965$. 
TALAIRACH J, SZIKLA G, TOURNOUX P, PROSALENTIS A, BORDAS-FERRER M, COVELLO J, JACOB M, MEMPEL H, SUSER P, BANCAUD J: Atlas d'Anatomie Stéréotaxique du Télencéphale. Masson \& Cie, Paris, 1967. 